\title{
Innovating dementia care; implementing characteristics of green care farms in other long-term care settings
}

\author{
Yvette Buist, ${ }^{1}$ Hilde Verbeek, ${ }^{2}$ Bram de Boer ${ }^{2}$ and Simone R. de Bruin ${ }^{1}$ \\ ${ }^{1}$ Centre for Nutrition, Prevention and Health Services, National Institute for Public Health and the Environment, BA Bilthoven, the Netherlands \\ ${ }^{2}$ Department of Health Services Research, CAPHRI Care and Public Health Research Institute, Faculty of Health, Medicine and Life Sciences, Maastricht \\ University, MD Maastricht, the Netherlands
}

ABSTRACT

Background: People with dementia at green care farms (GCFs) are physically more active, have more social interactions, are involved in a larger variety of activities, and come outdoors more often than those in other long-term dementia care settings. These aspects may positively affect health and well-being. This study explored which and how characteristics of GCFs could be implemented in other long-term dementia care settings, taking into account possible facilitators and barriers.

\begin{abstract}
Methods: Semi-structured interviews were conducted with 23 professionals from GCFs, independent smallscale long-term care facilities, and larger scale long-term care facilities in the Netherlands. The framework method was used to analyze the data.

Results: Several characteristics of GCFs (e.g. homelike aspects, domestic activities, and access to outdoor environments) have already been applied in other types of long-term dementia care settings. However, how and the extent to which these characteristics are being applied differ between GCFs and other types of longterm dementia care settings. Facilitators and barriers for the implementation of characteristics of GCFs were related to the physical environment in which the care facility is situated (e.g. the degree of urbanization), characteristics and competences of staff members (e.g. flexibility, creativity), characteristics and competences of managers (e.g. leadership, vision), and the political context (e.g. application of risk and safety protocols).

Conclusion: Several characteristics can be implemented in other dementia care settings. However, to realize innovation in dementia care it is important that not only the physical environment but also the social and organizational environments are supporting the process of change.
\end{abstract}

Key words: day programs, dementia, long-term care, nursing homes, qualitative research

\section{Introduction}

Green care farms (GCFs) offer innovative care services to people with dementia. GCFs provide care in small-scale, homelike environments in which agricultural activities are combined with care and support services for a variety of client groups, including people with dementia (De Bruin et al., 2009; Haubenhofer et al., 2010). At GCFs, everyday life is emphasized and people with dementia are encouraged to participate in meaningful activities that address their preferences, needs, and capacities. Such care is focused on

\footnotetext{
Correspondence should be addressed to: Simone R. de Bruin, PhD, National Institute for Public Health and the Environment, Centre for Nutrition, Prevention and Health Services, P.O. Box 1, 3720 BA Bilthoven, the Netherlands. Email: simone.de.bruin@rivm.nl. Received 19 Jul 2017; revision requested 4 Sep 2017; revised version received 8 Nov 2017; accepted 15 Nov 2017. First published online 16 January 2018.
}

enhancing the identity, autonomy, and social participation of people with dementia. Currently, GCFs are mainly present in Europe, especially in the Netherlands and Norway. In addition, outside Europe (e.g. Japan and the USA), the number of GCFs are gradually increasing. Most GCFs provide community-based services (De Bruin et al., 2009). In the Netherlands, for instance, approximately 200 GCFs are specialized in adult day services for people with dementia. New are GCFs providing 24-h nursing care, as an alternative for nursing homes (De Boer et al., 2015).

The delivery of care and support at GCFs is in line with a shift toward a more person-centred approach and small-scale care for people with dementia (Ausserhofer et al., 2016). In this new approach to health (care), health is not only seen as the absence of disease but rather emphasizes 
the "ability to adapt to the disease and to self-manage" (Huber et al., 2011). Focus is put on the ability of people with dementia to function in society according to his or her potential, to preserve autonomy, and to be involved in meaningful activities and social interaction (Dröes et al., 2016). Studies suggest that in current care delivery, preferences, needs, and capacities of people with dementia are insufficiently addressed. Recent studies, for instance, indicate that people with dementia feel isolated, lonely, compromised, and not at home in nursing homes (Knight and Mellor, 2007; Cahill and Diaz-Ponce, 2011). They spend large parts of their day inactive, immobile, and alone (Den Ouden et al., 2015). Studies into innovative dementia care concepts, such as smallscale, homelike care environments suggest that these concepts can positively affect outcomes (e.g. on the level of social engagement, social participation, and perceived autonomy) (Te Boekhorst et al., 2009).

A recent paper by De Bruin et al. (2017), introduced a framework describing valuable characteristics of GCFs, in which they distinguish themselves from other long-term care facilities, on different levels of the health system based on existing literature. On the micro level (i.e. the psychosocial environment including interactions with staff and other people with dementia), these characteristics included alignment of activities and services with preferences of people with dementia, integration of meaningful activities in everyday care, and freedom of choice. On the meso level (i.e. physical environment, including the activities offered and the organizational context including the vision on dementia care), these characteristics included a familiar- and smallscale environment, presence of animals and plants. Also, leadership in care vision was identified as a valuable characteristic since personal involvement by managers motivates staff. On the macro level (i.e. the context including funding, legislation, and quality assurance in long-term care), no differences between GCFs and other long-term care facilities were identified. The identified characteristics at the micro- and meso-levels of the health system are associated with improved outcomes in people with dementia (Clark et al., 2013; Fleming et al., 2016). Yet, it is unknown whether it might be possible to implement valuable characteristics of GCFs in other long-term settings. The aim of this study is to explore which valuable characteristics of GCFs can be implemented in other long-term care settings for people with dementia and how these characteristics could be implemented, taking into account possible facilitators and barriers. Longterm care facilities include settings that provide 24-h care, such as nursing homes and settings that provide adult day services for people with dementia.

\section{Methods}

A qualitative study was performed, in which data were collected between March and July 2016. Semi-structured interviews were conducted with professionals from three types of long-term care facilities in the Netherlands; GCFs delivering either adult day services and/or 24-h nursing home care, small-scale care facilities (e.g. small-scale living facilities), and larger-scale care facilities (e.g. nursing homes, regular adult day services centers) (see Table 1 for more detailed information about the different types of facilities). Additionally, two workshops (March and April 2017) were organized to present and discuss the findings from the interviews after these had been analyzed by our project team. Participants of the workshops were mostly professionals from GCFs and other long-term care facilities. Other participants were representatives of a healthcare insurance company and a regional association for professionals of GCFs and a family caregiver.

\section{Study sample}

For this study, purposive sampling (Mays and Pope, 1995) was used to ensure that all three types of long-term care facilities (see Table 1) were sufficiently reflected in our sample. To consider potential regional differences, we interviewed professionals from eight different provinces throughout the Netherlands. Representatives of the long-term care facilities were invited by email or by telephone to participate in the study. These facilities were either part of the network of the researchers involved in this study or were identified through an online search. If the professionals showed interest in participating, they were sent an additional information, including an informed consent form. For the interviews, 29 participants were approached (13 professionals of GCFs, 6 professionals of small-scale care facilities, and 10 professionals of larger-scale care facilities). In total, 23 participants agreed and were included in the study (12 professionals of GCFs, 5 professionals of small-scale care facilities, and 6 professionals of larger-scale care facilities). Reasons for nonparticipation were the change of position of the contact person, time restraints, and nonresponse. The interviewed professionals all had coordinating positions (e.g. manager, team leader, coordinator, lecturer practitioner, and innovation consultant). Furthermore, 30 people participated 
Table 1. Description of the different types of long-term care facilities included in this study

TYPE OF LONG-TERM CARE FACILITY DESCRIPTION

Green care farms (GCFs)

Farms providing care in small-scale, homelike environments in which agricultural activities are combined with care and support services for a variety of client groups, including people with dementia. People with dementia are encouraged to participate in a diverse range of meaningful and stimulating activities (e.g. picking eggs, preparing meals, gardening, sweeping the yard, feeding, and viewing the animals) centered around their normal daily life and integrated in a daily routine of the farm. GCFs generally have some degree of farming (i.e. crops, livestock, and woodland) and care (health, educational, and/or social services), but the ratio between farming and care, the type of farm (e.g. dairy farm, industrial livestock farm, and mixed farm), and the client groups differ. GCFs either provide adult day services and/or 24-h nursing care, as an alternative for nursing homes (De Bruin, 2009; De Boer et al., 2015)

Regular small-scale care facility

Small-scale care settings providing either adult day services and/or 24-h nursing home care in a homelike environment. Small-scale care facilities can be either stand-alone facilities in an urban neighborhood or in a more rural area. They can also be clustered on the grounds of a larger-scale nursing home. As GCFs, small-scale care facilities generally have an innovative approach to dementia care (Ausserhofer et al., 2016)

Regular larger-scale care facility

Larger-scale care settings, situated in the built-up area, providing either adult day services and/or 24-h nursing home care. As opposed to GCFs and regular small-scale care facilities, these long-term care facilities often have a large-scale character breathing out a more institutional atmosphere. Daily life is mainly determined by routines and rules of the organization. In general, larger-scale care facilities appear to have a more traditional or institutional approach to dementia care than GCFs and smaller-scale care facilities (Te Boekhorst et al., 2009; De Boer et al., 2015)

Table 2. Main topics of the interviews

\begin{tabular}{|c|c|}
\hline TOPIC & EXAMPLES OF QUESTIONS \\
\hline $\begin{array}{l}\text { Reflection on the characteristics of GCFs (De } \\
\text { Bruin et al., 2017) }\end{array}$ & $\begin{array}{l}\text { - Do you recognize the characteristics of GCFs (e.g. do you apply } \\
\text { them in your own context too?) }\end{array}$ \\
\hline $\begin{array}{l}\text { Facilitators and barriers for implementation of the } \\
\text { characteristics of GCFs }\end{array}$ & $\begin{array}{l}\text { - Which characteristics could be implemented in other long-term } \\
\text { care environments? } \\
\text { - What are (potential) barriers and facilitators for implementation of } \\
\text { characteristics of GCFs? }\end{array}$ \\
\hline
\end{tabular}

in the workshops of whom some $(n=8)$ also participated in the interviews.

\section{Data collection}

Some days ahead of the interview, the participants were asked to study the framework displaying the valuable characteristics of GCFs (De Bruin et al., 2017). The interviewers visited the respondents at their care facility. The interviews were guided by a semi-structured interview format. The interview schedule covered the topics displayed in Table 2 .

The semi-structured interview schedule was similar for representatives of the three types of care facilities (GCFs, small, and large-scale facilities). However, since the care context was different for the professionals there was a slight difference in the interview approach. For example, the professionals from GCFs were asked to relate to their own experiences with valuable characteristics of GCFs, while professionals from other long-term care settings were asked to discuss the way in which they expected the characteristics of GCFs could be implemented in other long-term care facilities.

Initially, two researchers conducted two pilot interviews together. In these pilot interviews, the clarity, relevance, and sequence of the questions were tested. Based on the outcomes of the pilot, the formulation and sequence of the questions were slightly adjusted. The interviews, including the introduction lasted about $1 \mathrm{~h}$. Interviews were conducted until the interviewers agreed that the 
point of data saturation was reached. This point was reached when no new themes emerged and when there was a high rate of recurrence of responses (Giacomini et al., 2000). The interviews were audiotaped with the interviewees' permission and transcribed verbatim. Two workshops were organized (one in the southern part of the Netherlands and one in the middle part of the Netherlands). Results from the interviews were presented and discussed in small-group discussion. During the workshops, stakeholders from long-term care organizations, municipality, family caregivers, regular long-term care, and managers of GCFs jointly discussed the valuable characteristics of GCFs and how these were applicable to other long-term care facilities.

\section{Data analysis}

The analysis of the interviews was based on the framework method (Gale et al., 2013). First, the interviews were transcribed and familiarization with the interview data took place. Second, an initial analytical framework was created by developing predetermined codes, derived from the interview format (i.e. deductive approach). The multilevel approach of Grol and Wensing (2004), discussed during the interviews, was used to classify the facilitators and barriers. The multilevel approach distinguishes facilitators and barriers at the following levels, facilitators or barriers related to: (i) the innovation itself, including environment and activities; (ii) the individual professional, i.e. all staff working in the different long-term care facilities such as (registered) nurses, nurse assistants, and nurse aides who in the Netherlands are mostly certified professionals who followed a specified education program of 2-3 years; (iii) the person with dementia; (iv) the social context; (v) the organizational context, and (vi) the economic and political context (Grol and Wensing, 2004). Subsequently, several interview transcripts were read, to complement the analytical framework by adding relevant and recurring codes (i.e. inductive approach). When no new themes emerged, all researchers involved discussed the analytical framework and agreed on the coding structure (Gale et al., 2013). The program MAXQDA 12 was used to enable coding the transcripts. After the coding process, the coded data were examined, recurring themes were defined and interpreted and drafts of the study findings were developed. During the process of analysis, drafts of the study findings were discussed by the authors. Draft study findings were additionally discussed in two workshops to validate findings through "member checking" (Giacomini et al., 2000). The participants were asked whether they understood the findings and whether the findings made sense from their point of view. Their feedback helped us to further refine our findings.

\section{Ethics statement}

In the Netherlands, the Medical Research Involving Human Subjects Act (WMO) does not apply to studies like the one described in this paper. Ethical approval therefore did not have to be obtained. All participants signed a written informed consent.

\section{Results}

The small-scale care facilities and larger-scale care facilities differed in their extent to which they were open to innovations in care and in implementing green aspects in their care delivery. First, we elaborate on which characteristics of GCFs could be implemented and why these would be valuable. Then, we discuss how these characteristics are or can be implemented in other long-term care settings.

\section{Characteristics of GCFs that can be implemented}

The interviews revealed that most of the characteristics of GCFs can be implemented in other longterm care facilities. In particular, three groups of characteristics were mentioned: characteristics of the psychosocial environment, characteristics of the activities offered, and characteristics of the physical environmental.

\section{PSYCHOSOCIAL ENVIRONMENT}

One of the characteristics of the psychosocial environment of GCFs is professionals' focus on the alignment of activities and care delivery with clients' personal preferences, needs, and capacities. Professionals of GCFs described the importance of paying attention to the different needs of people with dementia and the engagement of care professionals with the person with dementia. They mentioned that people with dementia should be able to decide for themselves how they want to spend their day. GCFs professionals highlighted the importance of ensuring that people with dementia feel understood and appreciated. There is also a strong focus on stimulating remaining capabilities of people with dementia.

That is what we see here. If you know the people very well, you know their potential, and what their capabilities are. You know their interests and what they can do well. And if you know this, then you can adjust the activities accordingly. For instance, that gentleman 
loves animals, for him, feeding a calf brings much more joy than shoveling in the garden. And if you offer the wrong activity, he will not like it. But you have to know someone to find out what he likes. (Manager GCF)

According to all interviewees, activities in all longterm care facilities can and should be aligned with personal preferences, needs, and capacities of people with dementia. In fact, most interviewees from small scale- and larger scale-care facilities noted that this has already been done in their care facility. Some professionals of small- and larger scale-care facilities described that people's personal preferences are their priority and standardized care comes at the second place. However, despite these preferred starting-points of the care delivery, it remained unclear from the interviews how alignment with preferences, needs, and capacities is being put in practice.

\section{Activities}

At GCFs, people with dementia are engaged in everyday and meaningful activities such as food preparation, cleaning windows, and sweeping the floor. Such activities are not specifically organized for people with dementia, but are often normal daily life activities and can as such emerge throughout the day. According to the GCF professionals, these activities can give people with dementia feelings of commitment and responsibility and can create feelings of reciprocity toward the environment. People with dementia are not only receiving care, but give something in return and are as such actively participating in the environment. This reciprocity encourages everyday life rather than emphasizing the medical aspects of the care facilities. Activities are meaningful and integrated in daily care routines. Additionally, the interviewed professionals suggested that such activities can encourage people with dementia to be physically and cognitively active which may positively affect their health.

In the backyard, we have made a chapel and a lot of the people like that. If you say to her: 'Can you please bring a candle to the chapel?', she is fine. But if we say: 'Maybe you can go for a walk', then she says: 'no way' $[. .$.$] So it [the activities] needs to have a purpose.$ And you can also bring the peels to the chickens, because the peels have to go to the chickens, and the underlying idea is that someone is physically active and goes for a walk. The chickens are far away, that's on purpose. But they don't know. It is not created, it's real. (Manager GCF)

Most professionals noted that the integration of meaningful and everyday activities could be implemented in other long-term care facilities. Professionals from some small- and larger-scale care facilities indicated that in their organizations, meaningful activities such as food preparation, site maintenance, and caring for plants and animals were already integrated in daily routines:

Every morning they know that they have to do something. In the morning, they are already waiting to check out the plants or the fruits, to water them and to feed the chickens. Every morning they have a purpose. And then you think: "Probably they don't realize it". In the morning they are standing in front of the door to do that. That is very special, that this is a trigger that they think: 'I have to do something today'. (Manager larger scale care facility)

Nevertheless, the interviews showed that there is often still a difference in approach toward activities between GCFs and other long-term care facilities. For example, this is the case for the extent to which meaningful activities are implemented. According to professionals from GCFs, meaningful activities include farming and domestic activities. According to professionals from small- and largerscale facilities, however, meaningful activities can also be recreational activities, such as watching movies, playing games, or attending beautician sessions. These are, in contrast to most farming and domestic activities, often pre-scheduled at specific points during the week.

Now, around this time, residents can go to the cinema, they can go for a beauty arrangement, pet animals. Well, residents could go for a walk, but that has been canceled, because of the bad weather. So at eleven they have four different options. That is today. And every day there is another program in which a variety of activities is being offered, and they can indicate themselves what they would like to do. (Manager larger scale care facility)

The interviews revealed that continuous presence of activities could be implemented by other longterm care facilities. This could be done by integrating well-being and care tasks of staff, meaning that staff integrates and combines care tasks with activities, for example, by involving people with dementia with household activities. Professionals of GCFs and professionals from small-scale care facilities suggested that integration of tasks could be reached by letting go restricted task descriptions of staff and thus by extending task descriptions. This implies that all staff members engage in a broad range of tasks in which flexibility is a facilitating feature. 


\section{PHYSICAL ENVIRONMENT}

Characteristics of the physical environment of GCFs include the homelike, lively, and activating environment with possibilities to go outdoors on a regular basis. Professionals of GCFs noted that people with dementia experience domestic stimuli and outdoor stimuli as valuable characteristics of GCFs. For example, indoors people with dementia experience stimuli concerning food preparation, while being outdoors they are stimulated by interacting with animals and people (e.g. veterinarian or milk truck). Moreover, some professionals of GCFs mentioned that the presence of animals encourages people with dementia to go outdoors. The animals have to be taken care of which can create a sense of urgency and motivate people to be active. As a manager of a GCF put it:

I think that being outside, the outdoor life, seeing movement, seeing other active people, that encourages being active, conversation, to reflect, to express meaning, wanting to tell stories. (Manager GCF)

Some interviewees of small- and larger scale-care facilities mentioned that they already implemented environmental characteristics such as a homelike environment and the presence of animals. All interviewees mentioned that most environmental characteristics such as access to different inand outdoor places, homelike environment, and presence of plants and/ or animals could be implemented by other long-term care facilities. In addition, the (care) environment could be reorganized to a more activating environment in which people with dementia experience everyday stimuli such as food preparation. Moreover, ensuring availability of multiple activities at different inand outdoor places in a care facility could further promote an activating environment. However, most interviewees mentioned that opening up outdoor places for people with dementia could not be done in all places; particularly in urban areas, this is perceived to cause safety risks.

\section{Facilitators and barriers to implement characteristics of GCFs}

Facilitators and barriers are distinguished at the following levels: the innovation (including GCFs environment and activities); the professional; people with dementia; social context; organizational context, and economic and political context (Grol and Wensing, 2004). Table 3 summarizes the facilitators and barriers to implement characteristics into other long-term care settings. It should be noted that the factors mentioned can be either facilitating or impeding. For example, the presence of innovative and anticipative staff members can be a facilitator in implementing innovations, while their absence can be a barrier. It should further be noted that the various facilitators and barriers are sometimes connected and/or could affect different aspects of care. For example, willingness and commitment of managers to implement changes may influence the extent to which care professionals dare to share innovative ideas but may also determine the design of physical environment.

\section{INNOVATION: ENVIRONMENT AND ACTIVITIES} OF GCFs

The interviewees noted that characteristics of the physical environment, such as the degree of urbanization and the available space in urban areas could be a barrier to implement characteristics of GCFs. At GCFs, there are different places (kitchen, stables, greenhouse, garden, workshop, etc.). In urban areas often, there is less space and fewer places are available. An urban environment can be dangerous for people with dementia to go outside (independently). Respondents indicated that people can get lost and can easily disappear from sight. Facilities located on an upper floor, which can be either adult day services centers or 24-h nursing home care facilities, reinforce the barrier to go outside. Although some interviewees noted that larger long-term care facilities in urban areas have plenty of space as long as doors to different wards are open. Hence, an open door policy is a facilitating factor. This enables people with dementia to walk around freely within the building.

Participant 1: Look, if they walk outside here, they are right on the highway. We really have to try not to let them go outside.

Participant 2: In the sense of openness, that is a limiting factor. There are limits in terms of space. [...]. However, because it is an open facility, in which you do not come across doors, it does not feel like a barrier for many people. People just go for a walk around, and then another round and again another round. It is not as in the past, when the doors were closed and people were really rattling at doors. (Manager larger scale care facility)

Another facilitating factor in offering a wide range of meaningful and everyday-life activities in longterm care facilities is that these activities are already present in the environment and no additional resources or materials have to be obtained.

INDIVIDUAL PROFESSIONAL

Innovative and anticipative staff members were mentioned by GCF professionals and professionals 
Table 3. Facilitators and barriers to implement characteristics of GCFs into other long-term care facilities

\begin{tabular}{|c|c|c|c|c|}
\hline $\begin{array}{l}\text { INNOVATION: } \\
\text { ENVIRONMENT AND } \\
\text { ACTIVITIES OF } \\
\text { GCFS }\end{array}$ & $\begin{array}{l}\text { INDIVIDUAL } \\
\text { PROFESSIONAL }\end{array}$ & $\begin{array}{l}\text { PEOPLE WITH } \\
\text { DEMENTIA }\end{array}$ & $\begin{array}{l}\text { SOCIAL AND } \\
\text { ORGANIZATIONAL CONTEXT }\end{array}$ & $\begin{array}{l}\text { ECONOMIC AND } \\
\text { POLITICAL } \\
\text { CONTEXT }\end{array}$ \\
\hline $\begin{array}{l}\text { Degree of } \\
\text { urbanization of the } \\
\text { area in which the } \\
\text { long-term care } \\
\text { facility is situated } \\
\text { and the available } \\
\text { space } \\
\text { - The floor on which } \\
\text { the adult day } \\
\text { services center/24-h } \\
\text { nursing care facility } \\
\text { is located } \\
\text { - The degree to which } \\
\text { activities are present } \\
\text { in the environment } \\
\text { The extent to which } \\
\text { additional materials } \\
\text { are needed for } \\
\text { activities }\end{array}$ & $\begin{array}{l}\text { - Innovativeness and } \\
\text { adaptability } \\
\text { - Presence of } \\
\text { innovative ideas and } \\
\text { a progressive } \\
\text { mindset } \\
\text { - Level of commitment } \\
\text { to people with } \\
\text { dementia } \\
\text { - Focus of care } \\
\text { (physical /medical care } \\
\text { vs. well-being) }\end{array}$ & $\begin{array}{l}\text { - Stage of } \\
\text { dementia }\end{array}$ & $\begin{array}{l}\text { - Willingness and commitment } \\
\text { of managers to implement } \\
\text { changes } \\
\text { - Time invested in supervising } \\
\text { activities } \\
\text { - Extent to which volunteers or } \\
\text { informal caregivers are } \\
\text { involved } \\
\text { - Flexibility of organizational } \\
\text { structure } \\
\text { - Level of engagement of } \\
\text { managers in nursing tasks } \\
\text { - Number of managers } \\
\text { - Role of staff members in } \\
\text { processes of change } \\
\text { - Training of staff members } \\
\text { - The type of policy regarding } \\
\text { freedom of movement of } \\
\text { people with dementia }\end{array}$ & $\begin{array}{l}\text { - Presence of } \\
\text { protocols } \\
\text { regarding risks } \\
\text { and safety }\end{array}$ \\
\hline
\end{tabular}

of other long-term care facilities as a facilitator in implementing characteristics of GCFs, such as meaningful activities and alignment of care with personal needs, in other care environments. They described that innovative and anticipative staff members can recognize impromptu opportunities to offer activities and to activate people with dementia. In addition, engagement of staff members was seen as a facilitator to ensure the alignment of care with personal need so that professionals can understand people with dementia by knowing the personal preferences. According to respondents, a barrier for implementing characteristics of GCFs was a focus on physical care rather than on wellbeing. Some GCF professionals and professionals of other long-term care facilities highlighted that they thought that more should be done to transition from a medical care perspective toward personcentered care. According to the GCF professionals and other professionals, the presence of staff with innovative ideas and a progressive mindset is a facilitating factor. These staff members could play an exemplary role, by taking the lead when stimulating people with dementia to use their capabilities.

\section{People With dementia}

Both, GCF professionals and other professionals noted that an advanced stage of dementia is a barrier to implementing certain characteristics of care farms, such as meaningful activities. The stage of the dementia was seen as a barrier in all three types of facilities. Dementia is a progressive disease and the deterioration will limit people's abilities to participate in everyday life. However, it was noted that people with limited abilities can and should still be able to enjoy petting animals, small walks, or a view for example.

...also walking in this area. But that is not possible for everyone due to mobility problems residents experience. However this garden is an option for everyone. (Manager small-scale care facility)

\section{SOCIAL AND ORGANIZATIONAL CONTEXT}

Most interviewees mentioned that the willingness of managers to implement changes is a major facilitating factor to implementing GCF characteristics. Some interviewees mentioned that the time it takes to supervise activities could be a barrier to provide (more) activities. However, it was also noted that this barrier could be dealt with by involving volunteers or informal caregivers to support the organization or supervision of activities. Nevertheless, some interviewees described that volunteers or informal caregivers cannot be left to their own devices. Volunteers also need coaching and guidance in providing care to the people with dementia, which also takes time.

So, it is very important to pay attention to the volunteers. Everyone can become a volunteer, but 
they have to be prepared. [...] Volunteers should get coaching by someone in the care network of the client. (Manager GCF)

A facilitator is to take organizational opportunities as a starting-point, for example, by offering activities and (re)designing the environment. This could enhance respect for client's lifestyles and preferences. Another factor that fosters change is the ensuring of a flexible organizational structure in which the needs and wishes of people with dementia are the starting-points. A flexible organizational structure could be a facilitator to implementing GCF characteristics such as the continuously and simultaneously presence of activities and to expose people with dementia to daily life stimuli. The physical environment could be adapted to promote this, although organizations have different ways of implementing this in practice, as the quotes below will illustrate.

A while ago, we had a bench in front of the door. And when the sun was shining staff members would go out to smoke a cigarette there. But then we noticed that they [people with dementia] become agitated and they want to go outside. They started tapping the window and pounding on the doors, because they also wanted to go outdoors to sit on the bench. So we removed the bench, since it only caused agitation. We concluded that the bench did not work because residents became agitated, and wanted to go outdoors, so we removed it. (Manager larger scale care facility)

I have also worked at a facility where we noticed that just sending people to their own room, or to a common area, did not work. But when we created a place to sit together at the entrance of the building, it did work. Because that is the place where many things are happening. That's what people are looking for. (Manager small-scale care facility)

GCF professionals and professionals from other care facilities mentioned that a limited number of managers is a facilitator for the implementation of changes. According to the respondents, limited numbers of managers and/or few levels of management in the organization imply less people wanting to give their opinion and approval. This will mostly speed up decision-making processes. Furthermore, engagement of managers in nursing tasks was also mentioned by GCF professionals and professionals from other care facilities to be a facilitator for the implementation of changes. GCF professionals and professionals of small-scale care facilities often combine their management tasks with nursing tasks. It was described that this enables easier and quicker communication with staff members and allows managers to more easily anticipate certain situations. Through involvement of managers on the shop floor, the managers are also familiar with the way their staff works and they can respond to it. As a result, managers can also ensure that their vision is implemented in practice.

And we know all the people. And I have direct contact with informal caregivers, the lines are very short. We [the management] participate, so the connection to the employees is close. This allows you to show exemplary behavior, you can promote your vision. People who make the policy are the people who know what they are talking about. (Manager small-scale care facility)

Another facilitator mentioned by the interviewees was to give staff members a clear role in processes of change. Respondents said that staff members should be supported to come up with solutions for challenges. They mentioned, for example, the difficulty people with dementia experience in going outside when there is a restricted area. According to the respondents, staff members would be more motivated to support the change or chosen solution if they are involved in the process. A barrier mentioned by some of the respondents was the training of staff members. It was described that the educational system focuses on medical care and person-centered care is mostly neglected.

Really, young girls of 18 who are saying: "You just went to the toilet. Stop it." Hear what you say. If you just have been drinking a lot, you also have to go to the toilet twice in an hour. And then you don't go for four hours. People are not unwilling when they are young, but they need a role model and the vision in care needs innovation. (Manager small-scale care facility)

\section{ECONOMIC AND POLITICAL CONTEXT}

Some professionals of GCFs and professionals from other long-term care facilities noted that some protocols regarding risks and safety (e.g. hazard analysis and critical control points protocol) could be a barrier to implement certain characteristics from care farms. According to these respondents, especially activities that contain some risks are also the activities that encourage people to be active and to feel alive. Activities such as cooking, caring for animals, and working with machinery can involve safety and health risks such as decreased hygiene, allergies, and the risk of falling. Although all longterm care facilities have to conform to the same rules and regulations, professionals of GCFs and managers of small-scale care facilities mentioned that they experienced during visits that largerscale care facilities have a more rigid approach to risk and safety concerns. One professional of 
a larger scale long-term care facility described that they sometimes felt impeded by protocols, for example, when creating a homelike atmosphere (safety risks) and when involving people with dementia in the food preparation (hygiene risks). Hence, the interviews revealed a tension between embracing risks as part of life and avoiding risks to ensure optimal safety. As mentioned previously, a flexible organizational approach toward the needs and wishes of people with dementia could facilitate implementation of GCF characteristics (e.g. adaptation of physical environment to promote continuous and simultaneous presence of activities and exposure to daily life stimuli) and could also be facilitated when dealing with protocols regarding risks and safety.

The respondents expressed that there are many changes in law and regulations concerning dementia care. Responsibility for adult day services has shifted from the national government to municipalities and therefore the procurement and financing of care have changed. However, these changes were not mentioned to be a facilitator or barrier to the implementation of GCF characteristics. Potentially requirements of municipalities could be either a facilitator or barrier depending on the vision of the municipality on dementia care.

I think, the system of an organization is also a barrier. So: "this is not possible because we can only order it this way, otherwise it cannot be imported in the system". Then you think: 'what a nonsense really'. So actually you need to make the organization inferior to living. (Manager larger scale care facility)

\section{Discussion}

This study found that valuable characteristics of GCFs, such as the psychosocial environment, activities, and physical environment could be implemented in other long-term care facilities. The presence of everyday-life activities, presence of professionals with innovative ideas and a progressive mindset and willingness of managers to implement changes were regarded as facilitators for implementation. Commitment and willingness to implement characteristics was a major theme at the level of the individual professionals and at the social and organizational context. Also, various barriers were mentioned, such as degree of urbanization of the area in which the long-term care facility is situated and the available space, the stage of dementia and time investment in supervising activities. The extent to which a barrier is expected or experienced as an actual barrier also depends on commitment and willingness of the social and organizational context to implement characteristics of GCFs. At GCFs, valuable characteristics were integrated in the care environment and as such were available throughout the day. At other longterm care facilities, however, such characteristics were often part of a program and are therefore only available at certain times. In order to actually integrate GCF characteristics, further innovation needs to be undertaken.

This study forms part of the developments concerning person-centeredness in dementia care (Love and Pinkowitz, 2013). Applying a personcentered approach can be beneficial for both people with dementia and professionals. For people with dementia, there are indications that a personcentered approach reduces anxiety, neuropsychiatric symptoms and depression, and might improve quality of life (Kim and Park, 2017). For care professionals, it is indicated that a person-centered approach in dementia care results in a reduction of staff stress, perceived hospitality and that it enables staff to provide care and activities they want to provide (Edvardsson et al., 2014). The results of this study show the importance of the physical care environment in promoting delivery of personcentered care. Other studies also show that it is important that the care environment is an open, homely place that supports people with dementia (Fleming et al., 2016; Chaudhury et al., 2017). Previous studies have highlighted that a sense of home in a nursing home is influenced by e.g. the building, engagement with others and activities, and having a connection with nature (Van Hoof et al., 2016). It has also been found that a sense of home is fragile and re-adjustable when life events occur (Soilemezi et al., 2017). That is why the preferences of people with dementia regarding their physical care environment should be considered. Several studies also underline our findings of the importance of leadership, professional vision, and engagement of managers in care practice as a role model in the implementation process of personcentered care (Rokstad et al., 2015; Backman et al., 2016). Committed managers, social support (i.e. meaning the support experienced by colleagues and managers), and sharing a vision can contribute to a positive psychosocial climate in which personcentered care can be implemented. It also motivates staff to achieve this vision (McCormack and McCance, 2011; Adams et al., 2017). In line with our study, it has also been suggested in earlier studies that care professionals should be involved in innovation in long-term care and that an important facilitator is a positive impact of these innovations on people with dementia (Rapaport et al., 2017). 


\section{Methodological considerations}

Professionals from innovative long-term care facilities may be overrepresented in our sample, as they were more open to being interviewed than managers from regular care settings. It could be that professionals who are already involved in innovations in care are more willing to share their experiences than professionals who work in a more conservative care environment. This may have resulted in a more positive attitude toward implementation of the elements in practice. However, we addressed this issue in the two workshops in which professionals from other longterm care facilities (i.e. professionals that were not interviewed) were present to reflect on our findings, to ensure different types of stakeholders understood the findings and that our findings made sense from different viewpoints.

Furthermore, we only conducted interviews and therefore we do not have any evidence on how elements were actually implemented in real-life everyday care and whether our respondents might have been more positive in their responses than in practice.

Also, we need to acknowledge that the professionals from regular long-term care institutions, may have given socially desirable answers, e.g. by indicating that valuable characteristics are already present in their own care settings. As a result, the care services may in practice have been less aligned with the needs, preferences, and capacities of people with dementia than they described. However, since the semi-structured interviews addressed topics from different angles, we aimed to prevent socially desirable answers as much as possible. Future studies could be conducted based on interviews with people with dementia and informal caregivers to find out if there is a discrepancy.

\section{Implications for practice, policy, and science}

Several countries are innovating current longterm care services and pay more and more attention to the characteristics of dementia care environments and the delivery of person-centered care (Day et al., 2000; De Bruin et al., 2017). Increasingly, studies (Anderiesen et al., 2014; Fleming et al., 2016) are addressing the importance of offering activities that are in line with the possibilities and capacities of the person with dementia and recommend reducing the focus on a person's potential limitations. Based on our study, we recommend that care organizations try to realize a supportive organizational environment, and not only focus on the interventions, physical environment, and activities. Thus, for example, not only establishing and/or opening gardens, but also making sure staff and management have a clear and shared vision on how to use these gardens in an optimal way.

Furthermore, the combination of the competencies of employees and leadership and vision of managers are essential to transfer elements of GCFs to other long-term care facilities for people with dementia. Therefore, managers of care facilities should encourage their employees to express and execute creative ideas and solutions. Managers often have a crucial role to implement vision in practice. It is, therefore, important that they actively set an example at the shop floor and show, for example, how to provide person-centered care. Also, coaching by managers and reflecting on actions by care professionals should be part of the implementation process.

In addition, mutual exchange of knowledge and experiences between various forms of long-term care settings is important. This exchange can be valuable to find a balance between provision of high quality medical/physical care, a person-centered approach, and promotion of well-being. Therefore, managers of GCFs and of other long-term care facilities are recommended to create opportunities for exchange, for example, by connecting care professionals from GCFs and other long-term care facilities and encourage them to accompany each other on a working day. Furthermore, collaboration between care facilities and universities or research institutes can enhance ground for design and implementation of innovations in dementia care. This also includes training of current and future healthcare professionals about healthcare innovations.

This study has also provided starting-points for further research. Although this study provided valuable insights in the extent to which valuable characteristics of GCFs are being implemented, detailed insights in what is the best way to put these in practice in regular settings is still lacking (Olsson et al., 2013; Rapaport et al., 2017). Also, there is still much unknown about how the characteristics, either in isolation or in combination, can affect outcomes in people with dementia. Further, studies focusing on these research questions are therefore desirable.

\section{Conclusion}

GCFs are seen as a valuable addition in dementia care of which several characteristics can be implemented in other dementia care settings as well. Facilitators and barriers for the implementation of characteristics of GCFs were 
related to the physical environment in which the care facility is situated (e.g. the degree of urbanization), characteristics and competences of staff members (e.g. flexibility, creativity, and ability to deliver person-centered care), characteristics and competences of managers (e.g. leadership, vision, and stimulating engagement of staff in innovating dementia care), and the political context (e.g. application of risk and safety protocols). However, to realize innovation in dementia care it is important that not only the physical environment but also the social and organizational environments are supporting the process of change.

\section{Conflict of interest}

None.

\section{Description of authors' roles}

Y. Buist collected and analyzed the data and wrote the paper. H. Verbeek designed the study, supervised data collection and analysis, and contributed to the paper. B. de Boer collected and analyzed data, and contributed to the paper. S. de Bruin designed the study, supervised data collection and analysis, and contributed to the paper. All authors read and approved the final version of the paper.

\section{Acknowledgments}

This work was supported by the Dutch Alzheimer Society and PGGM (Grant: WE.13-2014-04).

\section{References}

Adams, J., Verbeek, H. and Zwakhalen, S. M. (2017). The impact of organizational innovations in nursing homes on staff perceptions: a secondary data analysis. fournal of Nursing Scholarship, 49, 54-62.

Anderiesen, H., Scherder, E. J., Goossens, R. H. and Sonneveld, M. H. (2014). A systematic review-physical activity in dementia: the influence of the nursing home environment. Applied Ergonomics, 45, 1678-1686.

Ausserhofer, D. et al. (2016). "There's no place like home": a scoping review on the impact of homelike residential care models on resident-, family-, and staff-related outcomes. Fournal of the American Medical Directors Association, 17, 685-693.

Backman, A., Sjögren, K., Lindkvist, M., Lövheim, H. and Edvardsson, D. (2016). Towards person-centredness in aged care-exploring the impact of leadership. Fournal of Nursing Management, 24, 766-774.
Cahill, S. and Diaz-Ponce, A. M. (2011). 'I hate having nobody here. I'd like to know where they all are': can qualitative research detect differences in quality of life among nursing home residents with different levels of cognitive impairment? Aging \&o Mental Health, 15, 562-572.

Chaudhury, H., Cooke, H. A., Cowie, H. and Razaghi, L. (2017). The influence of the physical environment on residents with dementia in long-term care settings: a review of the empirical literature. The Gerontologist. Epub ahead of print. doi: 10.1093/geront/gnw259.

Clark, P., Mapes, N., Burt, J. and Preston, S. (2013). Greening Dementia - A Literature Review of the Benefits and Barriers Facing Individuals Living with Dementia in Accessing the Natural Environment and Local Green Space. Natural England Commissioned Reports, No.137. Worcester: Natural England and other parties.

Day, K., Carreon, D. and Stump, C. (2000). The therapeutic design of environments for people with dementia: a review of the empirical research. The Gerontologist, 40, 397-416.

De Boer, B., Hamers, J. P., Beerens, H. C., Zwakhalen, S. M., Tan, F. E. and Verbeek, H. (2015). Living at the farm, innovative nursing home care for people with dementia - study protocol of an observational longitudinal study. BMC Geriatrics, 15, 144.

De Bruin, S. R. (2009). Sowing in the Autumn SeasonExploring Benefits of Green Care Farms for Dementia Patients (Ph.D. thesis). Wageningen: Wageningen University.

De Bruin, S. R. et al. (2009). Green care farms promote activity among elderly people with dementia. Fournal of Housing for the Elderly, 23, 368-389.

De Bruin, S. R., De Boer, B., Beerens, H., Buist, Y. and Verbeek, H. (2017). Rethinking dementia care: the value of green care farming. Fournal of the American Medical Directors Association, 18, 200-203.

Den Ouden, M. et al. (2015). Daily (In) activities of nursing home residents in their wards: an observation study. Fournal of the American Medical Directors Association, 16, 963968.

Dröes, R. et al. (2016). Social health and dementia: a European consensus on the operationalization of the concept and directions for research and practice. Aging $\mathbb{E}$ Mental Health, 21, 1-14.

Edvardsson, D., Sandman, P. and Borell, L. (2014). Implementing national guidelines for person-centered care of people with dementia in residential aged care: effects on perceived person-centeredness, staff strain, and stress of conscience. International Psychogeriatrics, 26, 1171-1179.

Fleming, R., Goodenough, B., Low, L. F., Chenoweth, L. and Brodaty, H. (2016). The relationship between the quality of the built environment and the quality of life of people with dementia in residential care. Dementia, 15, 663-680.

Gale, N. K., Heath, G., Cameron, E., Rashid, S. and Redwood, S. (2013). Using the framework method for the analysis of qualitative data in multi-disciplinary health research. BMC Medical Research Methodology, 13, 117.

Giacomini, M. K., Cook, D. J. and Group, E.-B. M. W. (2000). Users' guides to the medical literature: XXIII. Qualitative research in health care A. Are the results of the 
study valid? Fournal of the American Medical Association, 284, 357-362.

Grol, R. and Wensing, M. (2004). What drives change? Barriers to and incentives for achieving evidence-based practice. Medical fournal of Australia, 180, S57-60.

Haubenhofer, D., Elings, M., Hassink, J. and Hine, R. (2010). The development of green care in western European countries. Explore, 6, 106-111.

Huber, M. et al. (2011). How should we define health? British Medical fournal, 343, d4163. doi: 10.1136/bmj.d4163.

Kim, S. K. and Park, M. (2017). Effectiveness of person-centered care on people with dementia: a systematic review and meta-analysis. Clinical Interventions in Aging, 12, 381-387.

Knight, T. and Mellor, D. (2007). Social inclusion of older adults in care: is it just a question of providing activities? International fournal of Qualitative Studies on Health and Well-being, 2, 76-85.

Love, K. and Pinkowitz, J. (2013). Person-centered care for people with dementia: a theoretical and conceptual framework. Generations, 37, 23-29.

Mays, N. and Pope, C. (1995). Rigour and qualitative research. British Medical fournal, 311, 109-112.

McCormack, B. and McCance, T. (2011). Person-Centred Nursing: Theory and Practice. Hoboken, NJ: John Wiley \& Sons.
Olsson, L. E., Jakobsson Ung, E., Swedberg, K. and Ekman, I. (2013). Efficacy of person-centred care as an intervention in controlled trials-a systematic review. fournal of Clinical Nursing, 22, 456-465.

Rapaport, P., Livingston, G., Murray, J., Mulla, A. and Cooper, C. (2017). Systematic review of the effective components of psychosocial interventions delivered by care home staff to people with dementia. British Medical fournal Open, 7, e014177.

Rokstad, A. M. M., Vatne, S., Engedal, K. and Selbæk, G. (2015). The role of leadership in the implementation of person-centred care using dementia care mapping: a study in three nursing homes. Fournal of Nursing Management, 23, 15-26.

Soilemezi, D., Drahota, A., Crossland, J., Stores, R. and Costall, A. (2017). Exploring the meaning of home for family caregivers of people with dementia. Fournal of Environmental Psychology, 51, 70-81.

Te Boekhorst, S., Depla, M. F., De Lange, J., Pot, A. M. and Eefsting, J. A. (2009). The effects of group living homes on older people with dementia: a comparison with traditional nursing home care. International fournal of Geriatric Psychiatry, 24, 970-978.

Van Hoof, J. et al. (2016). A three perspective study of the sense of home of nursing home residents: the views of residents, care professionals and relatives. British Medical fournal Geriatrics, 16, 169. 